BRAVILLIAN JOURNAL

www.bjournal.com.br
ISSN 0100-879X

Volume 43 (3) 182-267 March 2011

BIOMEDICAL SCIENCES

AND

CLINICAL INVESTIGATION

Braz J Med Biol Res, March 2011, Volume 44(3) 253-257

doi: 10.1590/S0100-879X2011007500006

Vancomycin-dependent Enterococcus faecium vanA: characterization of the first case isolated in a university hospital in Brazil

G. Kerbauy, M.R.E. Perugini, L.M. Yamauchi and S.F. Yamada-Ogatta

The Brazilian Journal of Medical and Biological Research is partially financed by

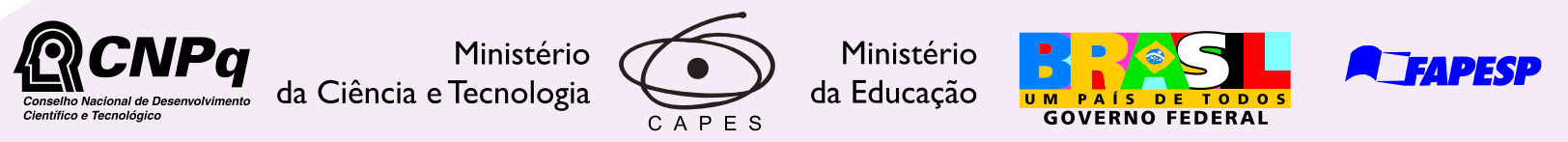

Institutional Sponsors

soripo
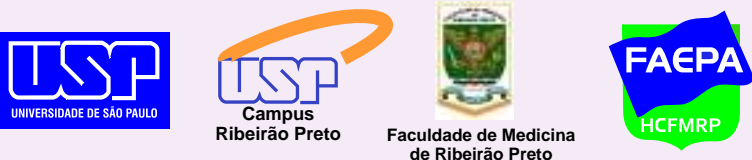

Ministério C A P E S $\oplus$ SHIMADZU Hotsite of proteomics metabolomics developped by:

GE Healthcare

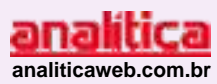




\title{
Vancomycin-dependent Enterococcus faecium vanA: characterization of the first case isolated in a university hospital in Brazil
}

\author{
G. Kerbauy ${ }^{1}$, M.R.E. Perugini ${ }^{2}$, L.M. Yamauchi ${ }^{1}$ and S.F. Yamada-Ogatta ${ }^{1}$ \\ ${ }^{1}$ Laboratório de Biologia Molecular de Microrganismos, Departamento de Microbiologia, \\ Centro de Ciências Biológicas, Universidade Estadual de Londrina, Londrina, PR, Brasil \\ ${ }^{2}$ Laboratório de Microbiologia Clínica, Departamento de Patologia, \\ Análises Clínicas e Toxicológicas, Centro de Ciências da Saúde, \\ Universidade Estadual de Londrina, Londrina, PR, Brasil
}

\begin{abstract}
In this study, we report the characterization of a strain of Enterococcus faecium vanA, which grows only in the presence of vancomycin (VDEfm-UEL). The bacterium was isolated from the feces of a female patient who had undergone surgical treatment of Reinke's edema and was receiving intravenous vancomycin therapy for infection with methicillin/oxacillin-resistant Staphylococcus aureus, a postoperative complication. Antimicrobial dependence was further confirmed by the vancomycin E-test. VDEfm-UEL was also shown to be resistant to ampicillin, ciprofloxacin, chloramphenicol, erythromycin, levofloxacin, penicillin, rifampicin, and teicoplanin. The putative virulence genes efaA, ge/E and esp were detected by PCR. The $d d l$ gene from VDEfm-UEL was cloned and sequenced. Vancomycin dependence seems to be associated with the insertion of a nucleotide in that sequence, which results in a frame-shift mutation, introducing a premature stop codon. This is the first report of vancomycin-dependent $E$. faecium isolation in a university hospital in Brazil.
\end{abstract}

Key words: Enterococcus faecium; Vancomycin; Dependence; vanA genotype; Virulence factors

\section{Introduction}

Over the last decades, infections due to glycopeptideresistant enterococci in healthcare-associated settings have been reported worldwide. Among enterococci, glycopeptide resistance is detected most commonly in Enterococcus faecium, which is often resistant to other classes of antibiotics $(1,2)$, and this feature has resulted in limited therapeutic options. Besides showing antimicrobial resistance, enterococci that require glycopeptides for growth have been isolated, particularly from patients on previous vancomycin therapy $(3,4)$.

We report here the isolation and characterization of vancomycin-dependent Enterococcus faecium (VDEfm) isolated from a rectal swab of a patient who had received prolonged intravenous vancomycin therapy for the treatment of methicillin/oxacillin-resistant Staphylococcus aureus (MRSA) infections. This is the first case of the isolation of VDEfm in Brazil, which occurred at the University Hospital of Londrina, Paraná, Brazil.

\section{Material and Methods}

\section{Case report}

A 65-year-old female patient was admitted to the University Hospital of Londrina, Paraná, Brazil, for surgical treatment of Reinke's edema. She stayed in a nursing home for preoperative analysis for 6 days. After the surgical procedure, she was transferred to the intensive care unit where she stayed for 4 days. Her clinical course was complicated by the development of meningitis and sepsis on the 5th postoperative day. MRSA was isolated from a blood culture and, on the basis of the antimicrobial susceptibility of the etiological agent, intravenous vancomycin (500 mg every $6 \mathrm{~h}$ ) and meropenen (2000 mg every $8 \mathrm{~h}$ ) were used for 22 days for the treatment of the infections. On the 16th day of antibiotic therapy, a rectal swab was taken from the patient as part of the hospital surveillance study for vancomycin-resistant enterococci (VRE). Colonies

Correspondence: S.F. Yamada-Ogatta, Departamento de Microbiologia, Centro de Ciências Biológicas, Universidade Estadual de Londrina, Rodovia Celso Garcia Cid, PR 445, km 380, 86051-980 Londrina, PR, Brasil. Fax: +55-43-3371-4788. E-mail: ogatta@uel.br

Received August 5, 2010. Accepted January 10, 2011. Available online January 21, 2011. Published March 7, 2011. 
growing on VRE agar (Oxoid, UK) supplemented with 6 $\mu \mathrm{g} / \mathrm{mL}$ vancomycin were identified as $E$. faecium, which was found to be growing only around the glycopeptideimpregnated discs in the agar diffusion assay (Figure 1A). The E-test further showed bacterial growth contiguous with the end of the strips containing the highest concentration of vancomycin (Figure 1B). The administration of antibiotics was discontinued because therapy was complete and a negative blood culture was confirmed. The patient was cured of infectious complications and left the hospital after 40 days of hospitalization. After 6 months, a rectal swab culture for VRE was negative, indicating that cessation of vancomycin had led to the clearance of VRE.

\section{Hospital, surveillance and microorganism isolation}

The University Hospital of Londrina is a 353-bed tertiary care center that serves the city of Londrina, in addition to several localities in the States of Paraná, São Paulo, and Mato Grosso do Sul. The intensive care center houses 35 patients distributed among 17 beds for general cases, 6 beds for burn cases, 7 beds for neonatal cases, and 5 beds for pediatric cases. Surveillance cultures of stools were examined weekly for all patients housed in intensive care units and for all patients found to be colonized by, or infected with VRE. A rectal swab was obtained from the patient and the sample was transferred to VRE broth (Oxoid) supplemented with $6 \mu \mathrm{g} / \mathrm{mL}$ vancomycin, $6 \mu \mathrm{g} / \mathrm{mL}$ ciprofloxacin and $8 \mu \mathrm{g} / \mathrm{mL}$ colistin. After a $18-\mathrm{h}$ incubation at $37^{\circ} \mathrm{C}$, a $100-\mu \mathrm{L}$ sample was spread on VRE agar supplemented with $6 \mu \mathrm{g} /$ $\mathrm{mL}$ vancomycin. The culture was further incubated at $37^{\circ} \mathrm{C}$ for $24 \mathrm{~h}$ under aerobic conditions. Bacteria were stored at $-80^{\circ} \mathrm{C}$ in $20 \%$ glycerol-brain heart infusion broth (Himedia, India) supplemented with $10 \mu \mathrm{g} / \mathrm{mL}$ vancomycin. The study protocol was approved by the Ethics Committee of the Universidade Estadual de Londrina (Protocol \#186-09/ CEP-UEL). The patient gave written informed consent to participate in the study.

\section{Phenotypic characterization}

Species identification was based on colony morphology, Gram stain, catalase assay, and the profile determined by the automated MicroScan WalkAway 96 Instrument (Dade MicroScan, USA). The disk diffusion method on Muller Hinton agar medium (Himedia) was carried out according to Clinical Laboratory Standard Institute (CLSI) (5) guidelines in order to determine the profile of antimicrobial susceptibility to linezolide $(30 \mu \mathrm{g})$, teicoplanin $(30 \mu \mathrm{g})$, tigecycline $(15 \mu \mathrm{g})$, and vancomycin $(30 \mu \mathrm{g})$. As bacterial growth was detected only around the glycopeptide-impregnated discs, the isolate was tested for susceptibility to 12 other antimicrobials (ampicillin, chloramphenicol, ciprofloxacin, erythromycin, gentamicin, levofloxacin, linezolide, penicillin, rifampicin, streptomycin, tetracycline, and tigecycline), using the automated broth microdilution panel of MicroScan WalkAway 96 according to manufacturer recommendations. The results reported here were those obtained after $24 \mathrm{~h}$ of incubation. The susceptibility breakpoints used were those recommended by the CLSI (5). E. faecalis ATCC 29212 and ATCC 51299 were used for quality control. The isolated bacterium was further tested by the vancomycin
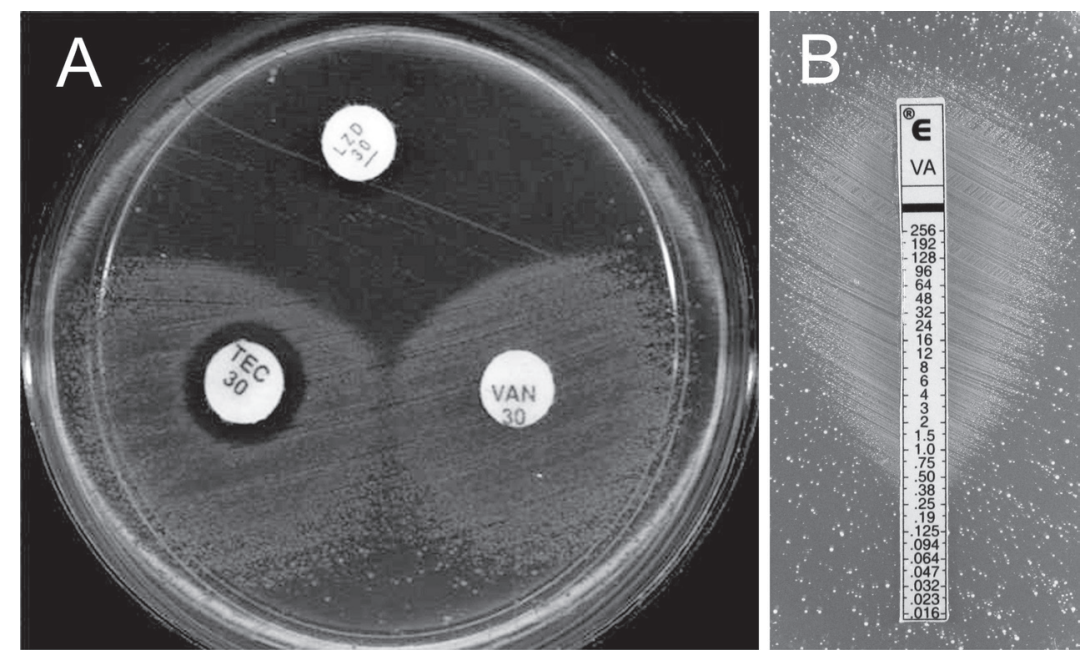

Figure 1. Growth dependence of vanA Enterococcus faecium UEL (VDEfm-UEL). A, Discs impregnated with $30 \mu \mathrm{g}$ linezolide (LZD), teicoplanin (TEC), and vancomycin (VAN) were placed on the surface of Muller Hinton agar plates previously inoculated with a suspension of VDEfm-UEL. After a $24-\mathrm{h}$ incubation at $37^{\circ} \mathrm{C}$, bacterial growth was observed only around the TEC and VAN discs. $B$, Vancomycin $E$-test showing the greater bacterial growth around the strips containing the highest concentration of the antimicrobial agent. 


\section{E-test (AB BIODISK, Sweden).}

\section{Genotypic characterization}

The vancomycin-resistance genotype and the putative virulence genes cyIA (activator of cytolysin, a secreted protein with hemolysin/bacteriocin activities), efaA (cell wall $E$. faecalis antigen $A$, an endocarditis-associated virulence factor), esp (enterococcal surface protein), and ge/E (gelatinase) were determined by PCR. The vancomycin resistance gene was determined using multiplex PCR as described by Petrich et al. (6). Genomic DNA of the enterococcal strain was extracted by the boiling method, and the virulence genes were determined as described by Ruzon et al. (7).

\section{Cloning and sequencing of the ddl gene}

The DNA fragments to be sequenced were amplified with the following primers based on the $d d l$ gene of $E$. faecium BM 4339 (8): forward 5' GAGTAAATCACTGAACGATT 3' and reverse 5' GGTTACGCAATCACTCCAGCCT 3'. PCR was performed in a final volume of $20 \mu \mathrm{L}$ containing $20 \mathrm{mM}$ Tris- $\mathrm{HCl}, \mathrm{pH} 8.4,50 \mathrm{mM} \mathrm{KCl}, 1.5 \mathrm{mM} \mathrm{MgCl}_{2}, 200$ $\mu \mathrm{M}$ of each dNTP, $10 \mathrm{pmol}$ of each primer, $2.5 \mathrm{U}$ Pfx DNA polymerase (Invitrogen, Brazil), and $10 \mu \mathrm{L}$ genomic DNA. The PCR product was purified from agarose gel with the QIAquick Gel Extraction kit (Qiagen, USA) and was inserted into the $\mathrm{pCR}^{\circledR} 2.1$ vector using the Original TA Cloning kit (Invitrogen) according to manufacturer recommendations. The insert was sequenced with a 3730xI DNA analyzer (Applied Biosystems, USA) using the Big Dye ${ }^{\circledR}$ Terminator v.3.1 Cycle Sequencing kit. A search for homologies in the GenBank/EMBL databases was carried out with the Blast algorithm (http://www.ncbi.nlm.nih.gov). The deduced amino acid sequence was analyzed with the ExPASy-Prosite program of the Swiss Institute of Bioinformatics, and the alignment of the sequences was carried out with ClustalW2 (http://www.cmbi.kun.nl/bioinf/tools/clustalw.shtml) of the EMBL-EBI software package.

\section{Results and Discussion}

The first VDE was isolated in 1994 from the urine of a female patient receiving long-term vancomycin therapy (3). Since then, other cases of VDE isolation from different clinical specimens of patients receiving previous vancomycin therapy have been reported (4). A vanA-type $E$. faecalis vancomycin-dependent strain from a non-human source was also reported by Tanimoto et al. (9). In that case, VDE was isolated from chicken meat imported from China. For the cases of VDE-infected/colonized patients, 7 were on vancomycin to treat non-enterococcal infections: 1 for diarrhea caused by Clostridium difficile, 5 for invasive staphylococcal infections, and 1 for bacteremia by Corynebacterium spp. resistant to ß-lactam antibiotics (4). In the remaining cases, VDE was considered to be the primary cause of infection.

In the present study, we report the first case of VDE isolation at a university hospital in Brazil (VDEfm-UEL). As reported in the other cases, the patient was receiving intravenous vancomycin therapy for non-enterococcal infection. This isolation was possible because of the protocol for detecting VRE in a selective culture medium supplemented with vancomycin, which is utilized in the infection prevention and control laboratory of the hospital. Besides requiring glycopeptides, the isolated bacterium was also resistant to ampicillin, ciprofloxacin, chloramphenicol, erythromycin, levofloxacin, penicillin, and rifampicin.

Although the isolated bacterium was considered to be colonizing Enterococcus according to CDC definitions of healthcare-associated infections (10), it harbored the putative virulence genes efaA, ge/E and esp, indicating a potential risk of infection. Low prevalence of the virulence markers has been shown for $E$. faecium isolates from different sources (11). In contrast to those results, we previously demonstrated a high prevalence of vancomycin-resistant $E$. faecium (VREfm) harboring virulence genes isolated from different sources at the University Hospital of Londrina, including the efaA, ge/E and esp genes (7). Camargo et al. (12) showed that the esp gene was restricted to VREfm isolates from the southern region of Brazil, which also harbored the hyaluronidase gene $(h y l)$. Studies with the E. faecalis efaA- mutant showed attenuation of virulence in a mouse peritonitis model when compared to the wildtype strain, suggesting that EfaA is a virulence factor (13). The chromosomal ge/E gene encodes an extracellular zinc metalloprotease that can participate in the translocation of bacteria across intestinal cell layers (14) and can contribute to bacterial virulence in a mouse peritonitis model (15). The presence of the esp gene, which encodes an enterococcal surface protein, has been associated with colonization and persistence of enterococci in ascending urinary tract infection in mice (16) and with biofilm formation on an abiotic surface (17).

Acquired vancomycin resistance in enterococci depends on the transcription of the van operon, leading to the production of the alternative structure D-alanine-D-lactate (D-Ala-D-Lac), instead of the dipeptide D-alanine-D-alanine (D-Ala-D-Ala) found in susceptible bacteria, where D-Ala$D$-Lac can be used as a cell wall constituent (18). On the other hand, vancomycin dependence has been associated with mutations in the $d d l$ gene, which codes for D-Ala-D-Ala ligase, an enzyme that plays a key role in cell wall biosynthesis $(4,8,9,19)$. In the absence of vancomycin, VDE is unable to synthesize either D-Ala-D-Ala or D-Ala-D-Lac, which could explain the glycopeptide dependence (19). A 1.15-kb fragment corresponding to the $d d l$ sequence of $E$. faecium vanA, which grows only in the presence of vancomycin (VDEfm-UEL) was cloned and sequenced. The nucleotide sequence of this fragment was deposited in GenBank with the accession No. HQ015715 and showed 98\% identity to 
Table 1. Point mutations in the ddl gene of vancomycin-dependent Enterococcus faecium.

\begin{tabular}{lcll}
\hline Mutation (BM4147 $\rightarrow$ VDEfm-UEL) & Position & Mechanism & Amino acid change \\
\hline GGC $\rightarrow$ AGC & 615 & Transition & Gly $\rightarrow$ Ser \\
$-\rightarrow$ A & 710 & Insertion & Frame-shift \\
TGT $\rightarrow$ CGT & 739 & Transition & Cys $\rightarrow$ Arg \\
C $\rightarrow-$ & 799 & Deletion & Frame-shift \\
GGA $\rightarrow$ GTA & 910 & Transversion & Gly $\rightarrow$ Val \\
ACG $\rightarrow$ CCG & 988 & Transversion & Thr $\rightarrow$ Pro \\
\hline
\end{tabular}

$(-)=$ absent.

the $d d l$ gene of $E$. faecium BM4147 (GenBank accession No. U39790). The alignment of the two nucleotide sequences showed several mutations in the $d d l$ gene of VDEfm-UEL compared to that of the VRE BM4147 strain, most of them being silent point mutations. The insertion of an adenine at nucleotide position 710 of the $d d l$ gene of VDEfm-UEL caused a frame-shift mutation, introducing a premature stop codon at position 733 of the sequence (Table 1) and thereby resulting in an inactive enzyme.

The isolation of an antibiotic-dependent strain alerts us to the importance of the control of healthcare-associated infections. This should be structured as an effective policy controlling the use of antibiotics in association with a laboratory capable of identifying microorganisms that show resistance to and also dependence on antibiotics.

\section{References}

1. Gales AC, Sader HS, Ribeiro J, Zoccoli C, Barth A, Pignatari AC. Antimicrobial susceptibility of Gram-positive bacteria isolated in Brazilian hospitals participating in the SENTRY Program (2005-2008). Braz J Infect Dis 2009; 13: 90-98.

2. Panesso D, Reyes J, Rincon S, Diaz L, Galloway-Pena $\mathrm{J}$, Zurita J, et al. Molecular epidemiology of vancomycinresistant Enterococcus faecium: a prospective, multicenter study in South American hospitals. J Clin Microbiol 2010; 48: 1562-1569.

3. Fraimow HS, Jungkind DL, Lander DW, Delso DR, Dean JL. Urinary tract infection with an Enterococcus faecalis isolate that requires vancomycin for growth. Ann Intern Med 1994; 121: 22-26.

4. Bert F, Leflon-Guibout V, Le Grand J, Bourdon N, NicolasChanoine $\mathrm{MH}$. [Emergence of vancomycin-dependent enterococci following glycopeptide therapy: case report and review]. Pathol Biol 2009; 57: 56-60.

5. CLSI - Clinical and Laboratory Standards Institute. Performance Standards for Antimicrobial Susceptibility Testing. Approved standard M100-S20-U. Wayne: CLSI; 2009.

6. Petrich AK, Luinstra KE, Groves D, Chernesky MA, Mahony JB. Direct detection of vanA and vanB genes in clinical specimens for rapid identification of vancomycin resistant
This can be reflected in the appropriate management of therapy and early adoption of precautionary measures to prevent the spread of infections by antibiotic resistant/ dependent microorganisms.

\section{Acknowledgments}

The present study was part of the M.Sc. dissertation of G. Kerbauy, who received a fellowship from CAPES. We thank Dr. A. Leyva for English editing of the manuscript, and Ediel Clementino da Costa and Jussevania Pereira Santos for technical support. Research supported by grants from Pro-Reitoria de Pesquisa e Pós-Graduação (PROPPG) of Universidade Estadual de Londrina (UEL).

enterococci (VRE) using multiplex PCR. Mol Cell Probes 1999; 13: 275-281.

7. Ruzon FI, Paula SB, Kanoshiki RL, Pereira-Santos J, Kerbauy G, Kobayashi RKT, et al. Virulence determinants in vancomycin-resistant Enterococcus faecium isolated from different sources at University Hospital of Londrina, Paraná, Brazil. J Microbiol 2010; 48: 814-821.

8. Gholizadeh Y, Prevost M, Van Bambeke F, Casadewall B, Tulkens PM, Courvalin P. Sequencing of the ddl gene and modeling of the mutated D-alanine:D-alanine ligase in glycopeptide-dependent strains of Enterococcus faecium. Protein Sci 2001; 10: 836-844.

9. Tanimoto K, Nomura T, Hamatani H, Xiao YH, Ike Y. A vancomycin-dependent VanA-type Enterococcus faecalis strain isolated in Japan from chicken imported from China. Lett Appl Microbiol 2005; 41: 157-162.

10. Horan TC, Andrus M, Dudeck MA. CDC/NHSN surveillance definition of health care-associated infection and criteria for specific types of infections in the acute care setting. $A m \mathrm{~J}$ Infect Control 2008; 36: 309-332.

11. Vankerckhoven V, Huys G, Vancanneyt M, Snauwaert C, Swings J, Klare I, et al. Genotypic diversity, antimicrobial resistance, and virulence factors of human isolates and 
probiotic cultures constituting two intraspecific groups of Enterococcus faecium isolates. Appl Environ Microbiol 2008; 74: 4247-4255.

12. Camargo IL, Gilmore MS, Darini AL. Multilocus sequence typing and analysis of putative virulence factors in vancomycin-resistant and vancomycin-sensitive Enterococcus faecium isolates from Brazil. Clin Microbiol Infect 2006; 12: 1123-1130.

13. Singh $\mathrm{KV}$, Coque TM, Weinstock GM, Murray BE. In vivo testing of an Enterococcus faecalis efaA mutant and use of efaA homologs for species identification. FEMS Immunol Med Microbiol 1998; 21: 323-331.

14. Zeng J, Teng F, Murray BE. Gelatinase is important for translocation of Enterococcus faecalis across polarized human enterocyte-like T84 cells. Infect Immun 2005; 73: 1606-1612.
15. Singh KV, Qin X, Weinstock GM, Murray BE. Generation and testing of mutants of Enterococcus faecalis in a mouse peritonitis model. J Infect Dis 1998; 178: 1416-1420.

16. Shankar N, Lockatell CV, Baghdayan AS, Drachenberg C, Gilmore MS, Johnson DE. Role of Enterococcus faecalis surface protein Esp in the pathogenesis of ascending urinary tract infection. Infect Immun 2001; 69: 4366-4372.

17. Heikens E, Bonten MJ, Willems RJ. Enterococcal surface protein Esp is important for biofilm formation of Enterococcus faecium E1162. J Bacteriol 2007; 189: 8233-8240.

18. Courvalin P. Vancomycin resistance in Gram-positive cocci. Clin Infect Dis 2006; 42 (Suppl 1): S25-S34.

19. Van Bambeke F, Chauvel M, Reynolds PE, Fraimow HS, Courvalin P. Vancomycin-dependent Enterococcus faecalis clinical isolates and revertant mutants. Antimicrob Agents Chemother 1999; 43: 41-47. 